\title{
Evaluation of Alternative Altitude Scaling Methods for Thermal Ice Protection System in NASA Icing Research Tunnel
}

\author{
Sam Lee \\ Vantage Partners, LLC \\ Harold E. Addy, Jr., retired \\ Andy P. Broeren \\ NASA Glenn Research Center \\ David M. Orchard \\ National Research Council Canada
}




\section{Outline}

- Introduction

- Experimental Methods

- Results and Discussion

- Conclusion 


\section{Introduction Motivation for Study}

- Most thermal Ice Protection System (IPS) development and testing done in atmospheric icing tunnels that cannot simulate altitude effects.

- Altitude scaling is required to test IPS in atmospheric wind tunnels.

- Re-based scaling methods with empirical corrections typically used.

- A more robust scaling methods desired for development of current and future generation aircraft

- Joint NASA and NRCC research program conducted to study the issue. 


\section{Introduction \\ NRC AIWT Tests (2012, 2014, 2015)}

- 18" chord NACA 0018 model with simple heated air IPS

- Re-based scaling method found to be inadequate

- Airfoil surface temperatures well matched between altitude and ground conditions.

- Accreted ice mass much larger than reference

- Ice formed farther downstream than reference

- Visual evidence of water re-entrainment into air flow.

- Two Weber number based method for scaling investigated.

- One method matched $\mathrm{We}_{\mathrm{DW}}$ (water density based)

- Other method matched We $e_{D A}$ (air density based) and $\mathrm{m}_{\mathrm{w}} / \mathrm{m}_{\mathrm{e}}$ (defined as $\mathrm{Pi} 3$ ). 


\section{Introduction \\ AIWT Tests $(2012,2014,2015)$}

- We-based scaling methods produced ice accretions much closer in size and location to altitude reference conditions than Re-based scaling method.

- Provided means of altitude scaling based on primary physics and not empirical corrections.

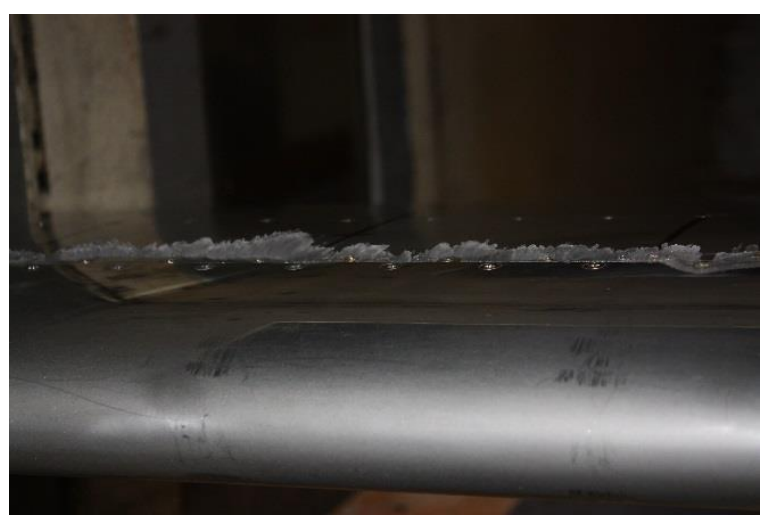

Reference

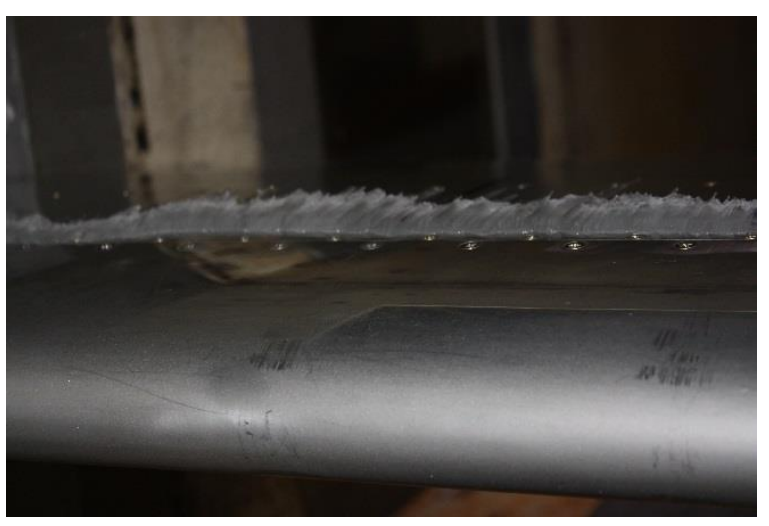

Re Scaling

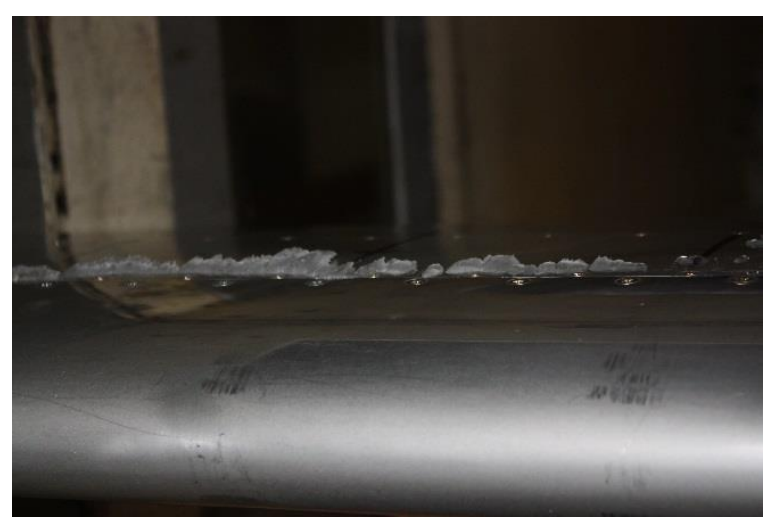

WePi3 Scaling 


\section{Introduction 2016 IRT Test}

- Compared different scaling methods with a much larger business jet airfoil model.

- Ice protection system more similar to what is used on commercial aircraft.

- IRT cannot simulate altitude conditions.

- Results of different scaling methods can be compared to one another and trends compared to AIWT results. 


\section{Experimental Procedure}

- Test conducted in lcing Research Tunnel at NASA Glenn Research Center.

- 60 in. chord model representative of modern business jet.

- Piccolo tube IPS.

- Built for 2006 Wichita State University IPS analysis and modelling study.

- Extensively instrumented with

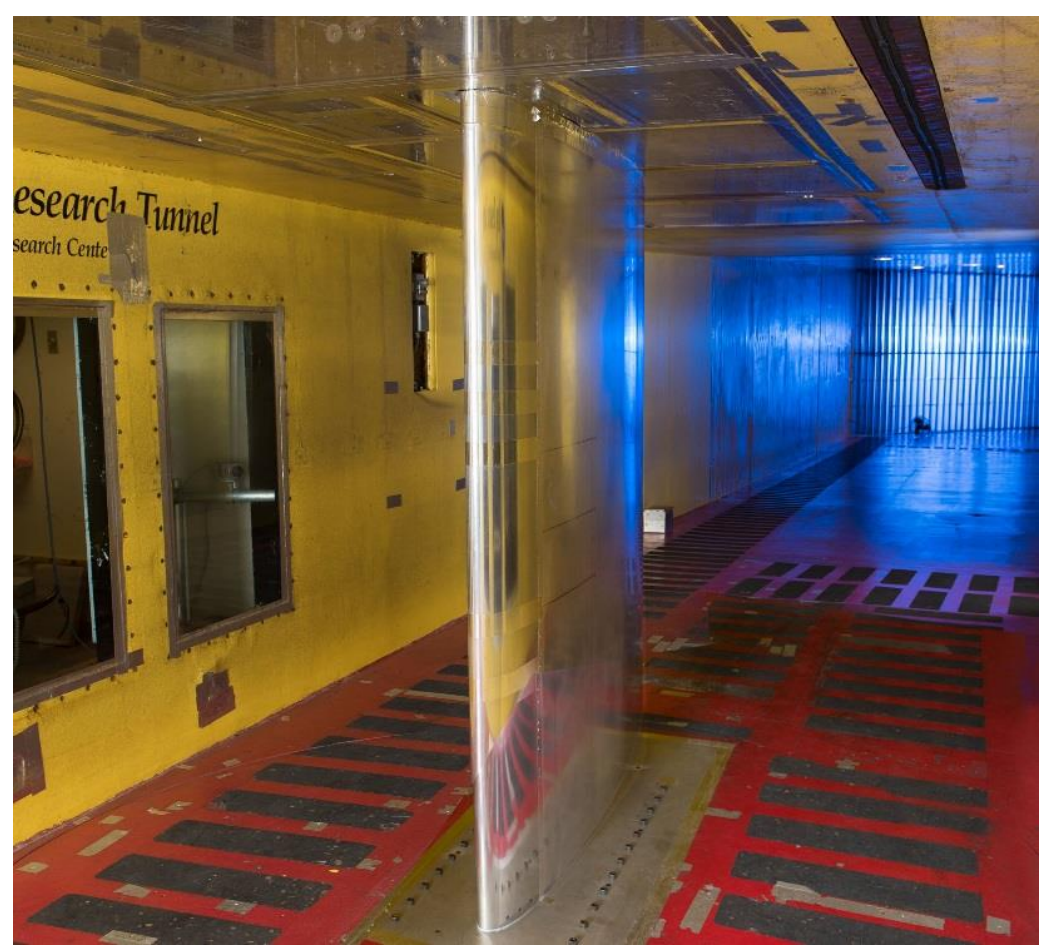
temperature and pressure sensors.

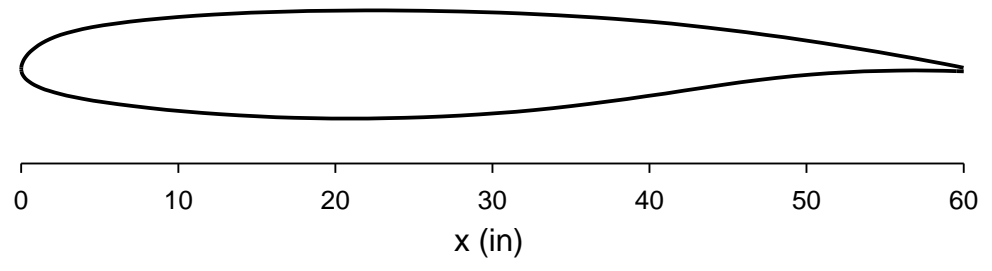




\section{Experimental Procedure}

- Surface temperatures (32 TCs)

- 4 TCs in piccolo tubes (Inlet T)

- 4 TCs in diffuser liner (Outlet T)
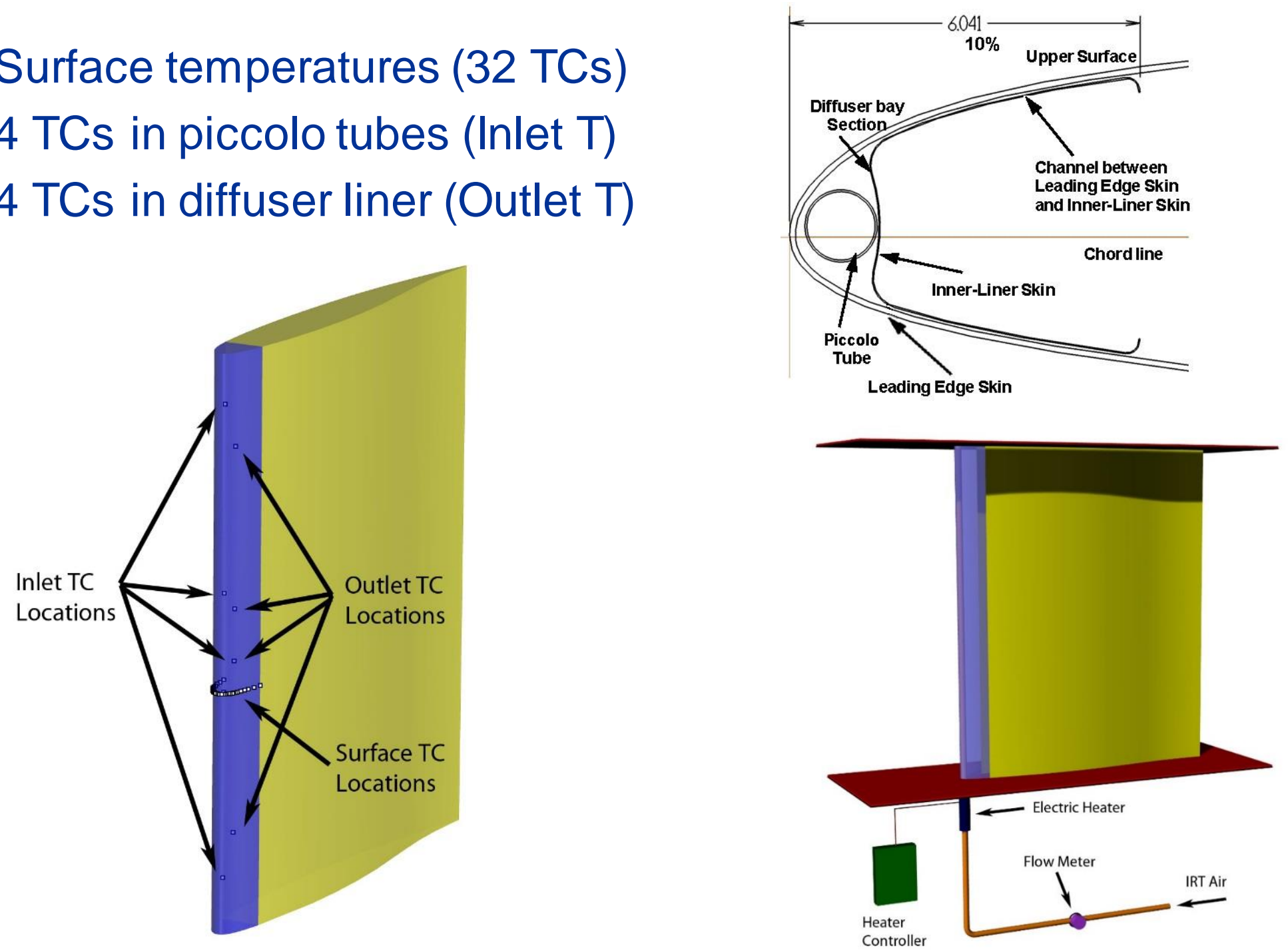


\section{Reference Conditions}

\begin{tabular}{|cccccccc|}
\hline Flight Phase & $\begin{array}{c}\text { Altitude } \\
(\mathrm{ft})\end{array}$ & $\begin{array}{c}\mathrm{V} \\
(\mathrm{kts})\end{array}$ & $\begin{array}{c}\alpha \\
(\mathrm{deg})\end{array}$ & $\begin{array}{c}\mathrm{T}_{\mathrm{s}} \\
(\text { deg C) }\end{array}$ & $\begin{array}{c}\mathrm{T}_{\mathrm{t}} \\
(\text { deg C) }\end{array}$ & $\begin{array}{c}\text { LWC } \\
\left(\mathrm{g} / \mathrm{m}^{2}\right)\end{array}$ & $\begin{array}{c}\text { MVD } \\
(\mu \mathrm{m})\end{array}$ \\
\hline Descent & 10000 & 180 & -1 & -14.2 & -10 & 0.35 & 19.1 \\
Cold Hold & 15000 & 180 & 3 & -20.1 & -15.8 & 0.24 & 17.5 \\
Warm Hold & 15000 & 180 & 3 & -8.6 & -4.3 & 0.49 & 17.4 \\
\hline
\end{tabular}




\section{Scaling Parameters}

\begin{tabular}{|c|c|c|c|c|}
\hline Scaling Method & \multicolumn{4}{|c|}{ Scaling Parameters Held Constant } \\
\hline $\operatorname{Re}$ & $\operatorname{Re}_{2 r}$ & $\mathrm{~m}_{\mathrm{w}}$ & $\overline{\mathrm{K}_{0}}$ & $\overline{T_{r}}$ \\
\hline WePi3 & WeDA & $\mathrm{Pi} 3$ & $\mathrm{~K}_{0}$ & $\mathrm{~T}_{\mathrm{r}}$ \\
\hline WeDW & WeDW & $m_{w}$ & $\mathrm{~K}_{0}$ & $T_{r}$ \\
\hline
\end{tabular}

- Required 2 step process to obtain scaled conditions

- Run at Re-scaled conditions to obtain L.E. temperatures.

- Run at We-scaled conditions with IPS adjusted to match the L.E. temperatures obtained at Re-scaled conditions. 


\section{Descent Scenario}

LWC MV $m_{N} \mathrm{Re}_{2}$

\begin{tabular}{ccccccccccccc}
$\begin{array}{c}\text { Scale } \\
\text { Method }\end{array}$ & Alt (ft) & $\mathrm{V}(\mathrm{kts})$ & $\begin{array}{c}\mathrm{T}_{\mathrm{s}} \\
(\operatorname{deg} \mathrm{C})\end{array}$ & $\begin{array}{c}\mathrm{LWC} \\
\left(\mathrm{g} / \mathrm{m}^{3}\right)\end{array}$ & $\begin{array}{c}\mathrm{MVD} \\
(\mu \mathrm{m})\end{array}$ & $\begin{array}{c}\mathrm{m}_{\mathrm{w}} \\
\left(\mathrm{g} / \mathrm{m}^{2} \mathrm{~s}\right)\end{array}$ & $\begin{array}{c}\mathrm{Re}_{2 \mathrm{r}} \\
\left(\mathrm{x} 10^{6}\right)\end{array}$ & WeDA & $\begin{array}{c}\text { Pi3 } \\
\left(\times 10^{6}\right)\end{array}$ & $\begin{array}{c}\text { WeDW } \\
(\mathrm{g})\end{array}$ \\
\hline Referenc & 10000 & 180 & -14.2 & 0.35 & 19.1 & 17.6 & 0.224 & 5814 & 1.6 & 6.21 & N/A \\
Re & 1066 & 133 & -12.7 & 0.48 & 22.8 & 17.6 & 0.224 & 4315 & 2.24 & 3.38 & 20 & 8.5 \\
WePi3 & 1439 & 159 & -13.5 & 0.35 & 19.4 & 14.4 & 0.265 & 5814 & 1.62 & 4.84 & 8.5 \\
WeDW & 1782 & 180 & -14.2 & 0.34 & 21.6 & 17.6 & 0.297 & 7769 & 1.88 & 6.21 & 12.7 \\
\hline
\end{tabular}

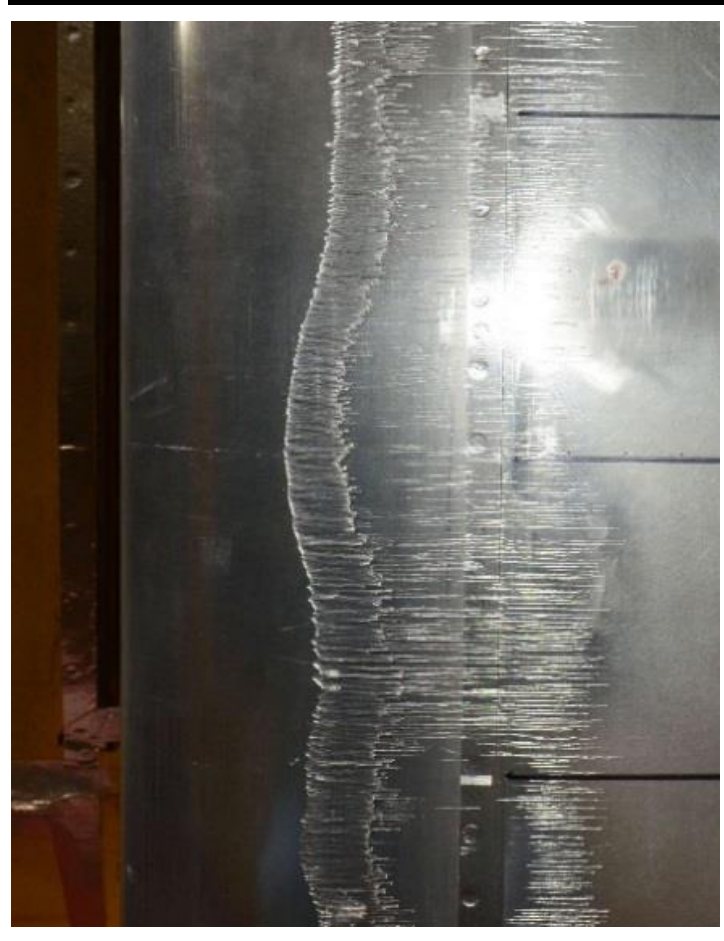

Re Scaling

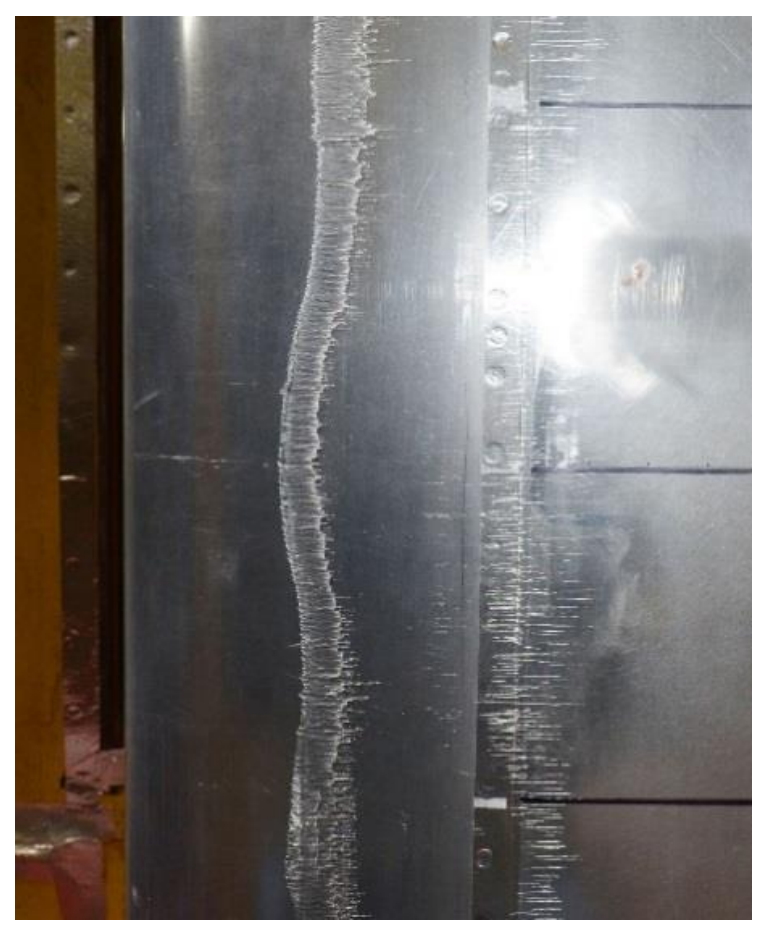

WePi3 Scaling

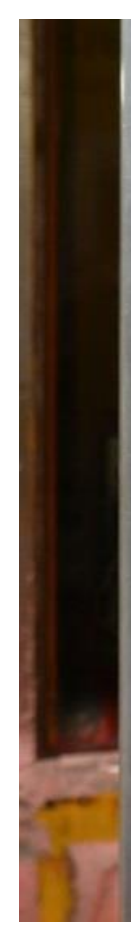

WeDW Scaling 


\section{Descent Scenario}

\section{LE Surface Temperature}

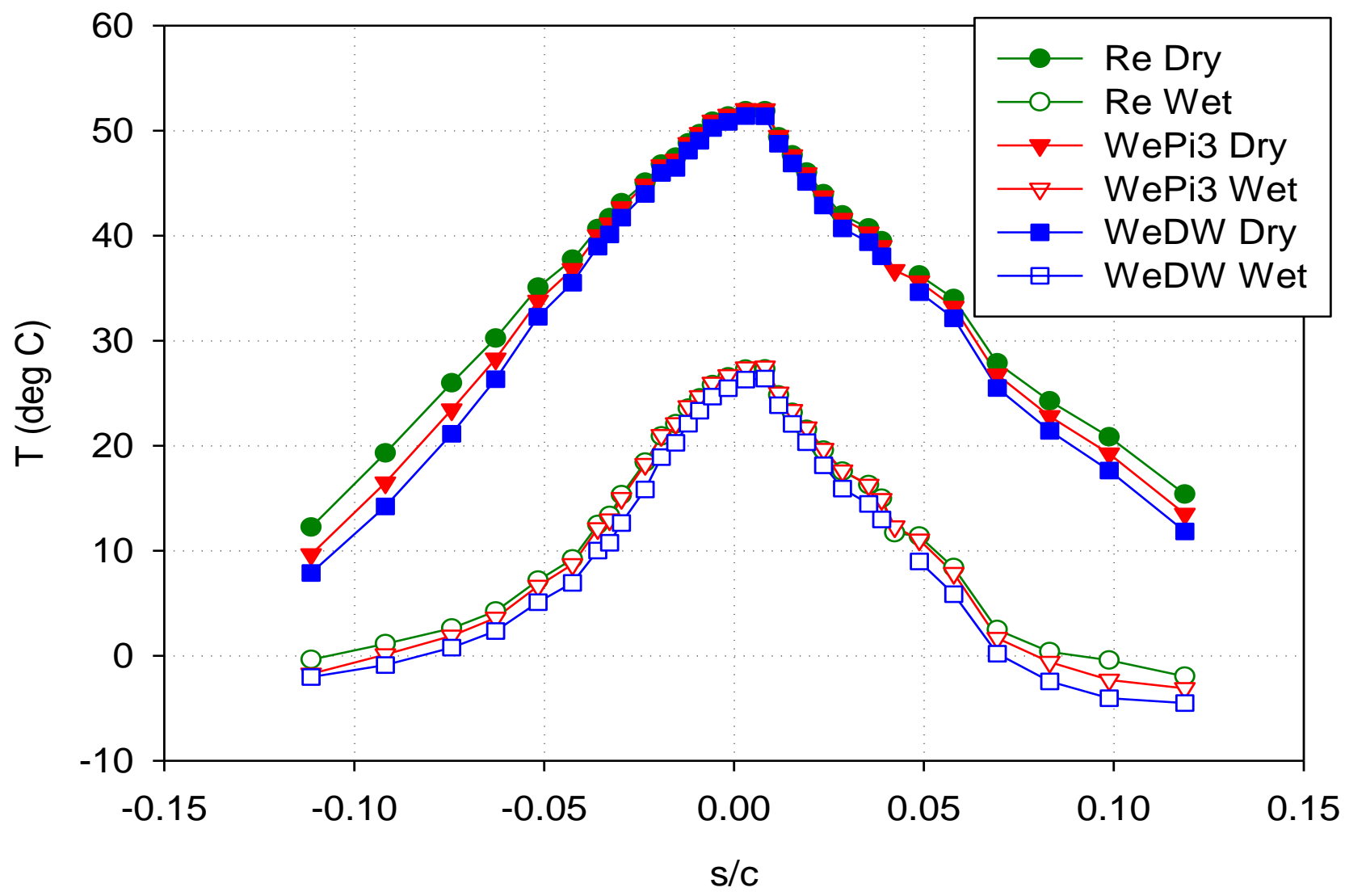




\section{Descent Scenario}

Heated Air Temp.

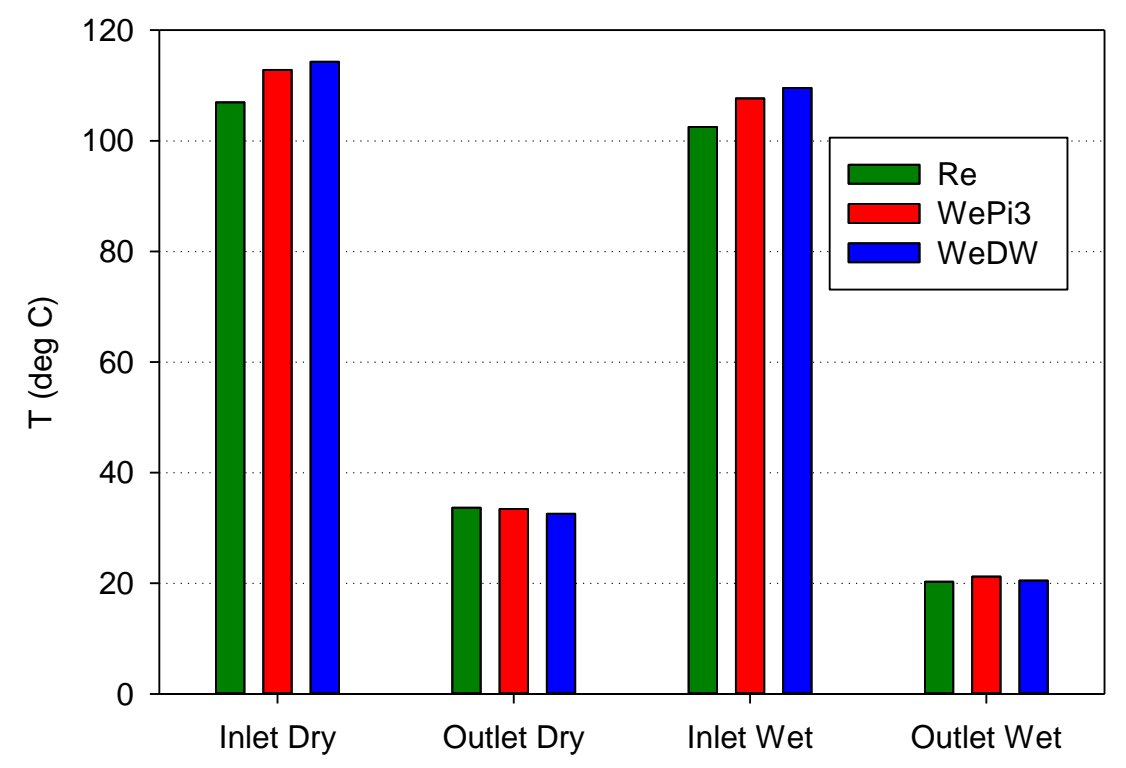

Heated Air Energy Input

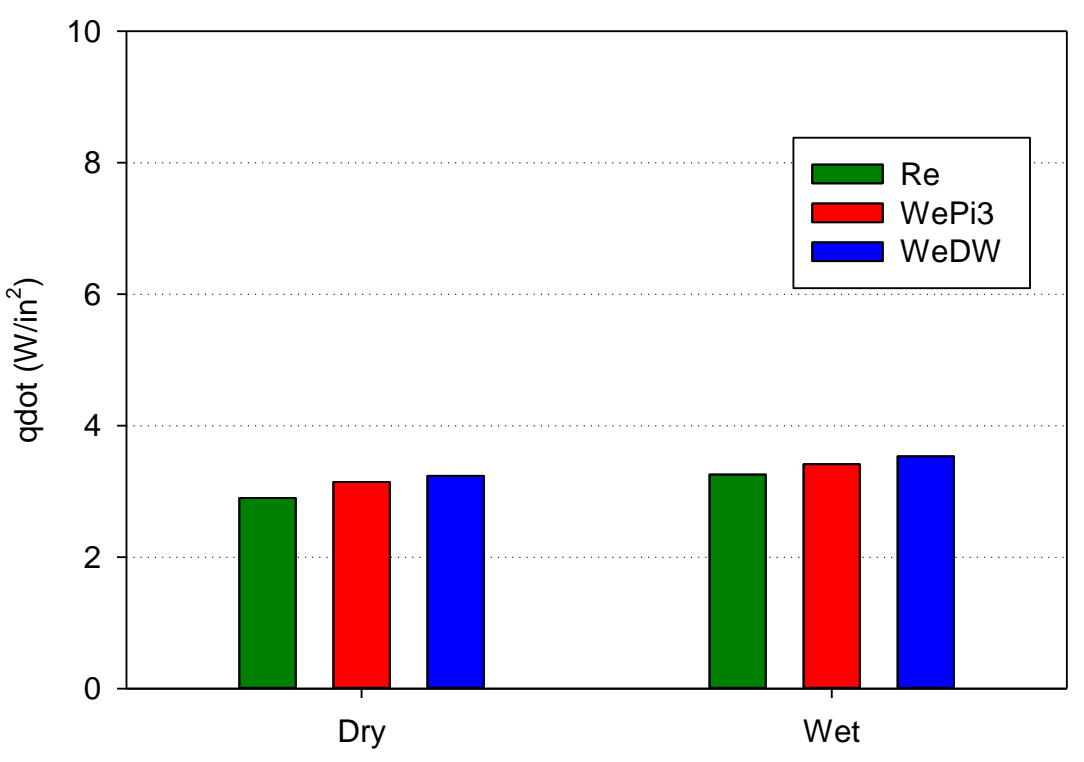




\section{Cold Hold Scenario}

\begin{tabular}{|cccccccccccc|}
\hline $\begin{array}{c}\text { Scale } \\
\text { Method }\end{array}$ & Alt $(\mathrm{ft})$ & $\mathrm{V}(\mathrm{kts})$ & $\begin{array}{c}\mathrm{T}_{\mathrm{s}} \\
(\mathrm{deg} \mathrm{C})\end{array}$ & $\begin{array}{c}\mathrm{LWC} \\
\left(\mathrm{g} / \mathrm{m}^{3}\right)\end{array}$ & $\begin{array}{c}\mathrm{MVD} \\
(\mu \mathrm{m})\end{array}$ & $\begin{array}{c}\mathrm{m}_{\mathrm{w}} \\
\left(\mathrm{g} / \mathrm{m}^{2} \mathrm{~s}\right)\end{array}$ & $\begin{array}{c}\mathrm{Re}_{2 \mathrm{r}} \\
\left(\mathrm{x} 10^{6}\right)\end{array}$ & WeDA & Pi3 & $\begin{array}{c}\text { WeDW } \\
\left(\mathrm{x} 10^{6}\right)\end{array}$ & $\begin{array}{c}\text { Ice Mass } \\
(\mathrm{g})\end{array}$ \\
\hline Reference & 15000 & 185 & -20.1 & 0.31 & 14.6 & 13.4 & 0.193 & 5147 & 1.06 & 6.54 & $\mathrm{~N} / \mathrm{A}$ \\
$\mathrm{Re}$ & 976 & 109 & -16.4 & 0.52 & 20.1 & 13.4 & 0.193 & 3065 & 1.87 & 2.27 & 54.2 \\
WePi3 & 1495 & 149 & -16 & 0.28 & 16.9 & 9.5 & 0.253 & 5147 & 1.06 & 4.22 & 5.0 \\
WeDW & 2087 & 185 & -15.7 & 0.27 & 18.9 & 13.3 & 0.31 & 8346 & 1.37 & 6.54 & $16.4^{*}$ \\
\hline
\end{tabular}

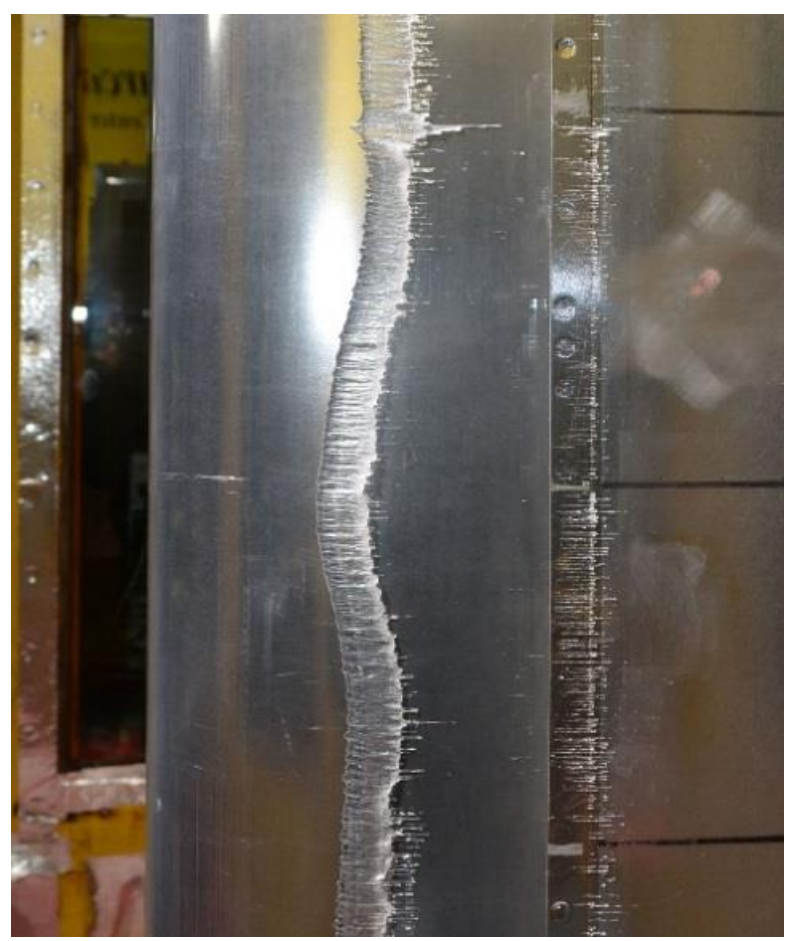

Re Scaling

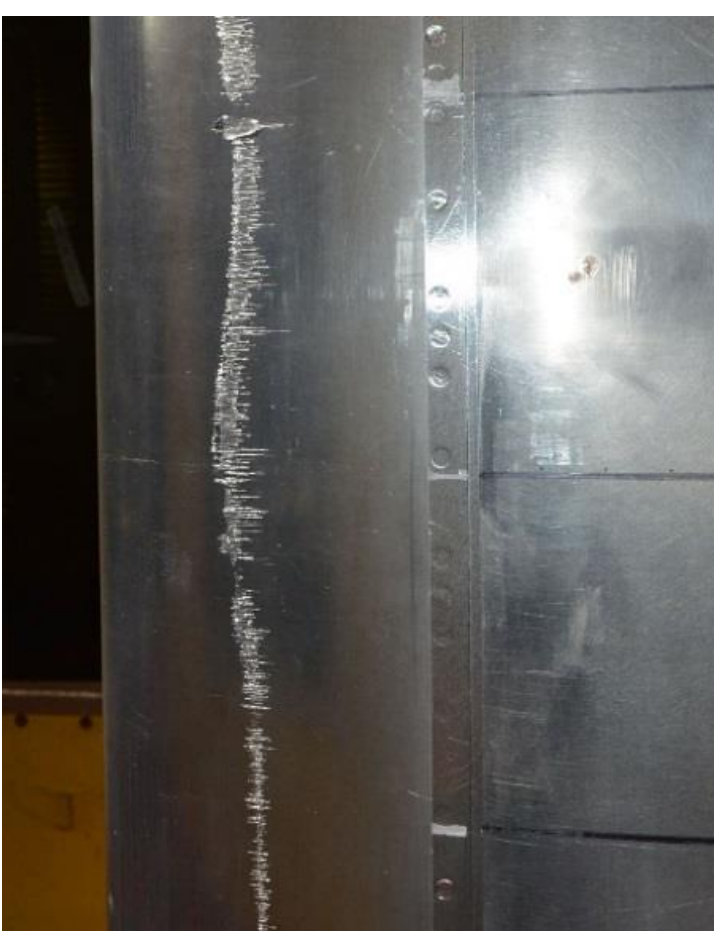

WePi3 Scaling

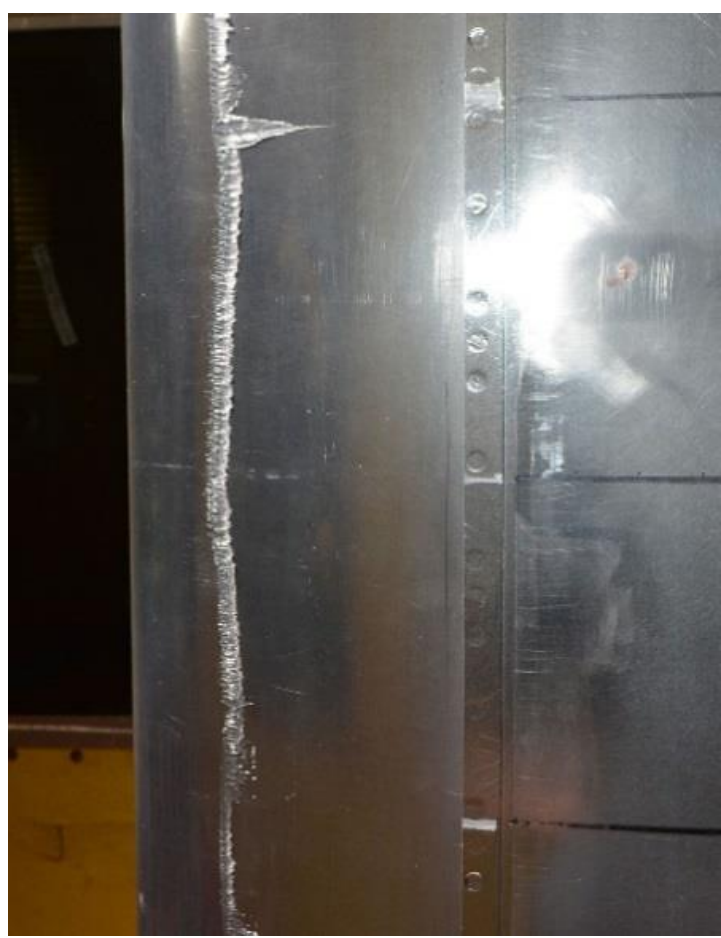

WeDW Scaling 


\section{Cold Hold Scenario}






\section{Cold Hold Scenario}

Heated Air Temp.

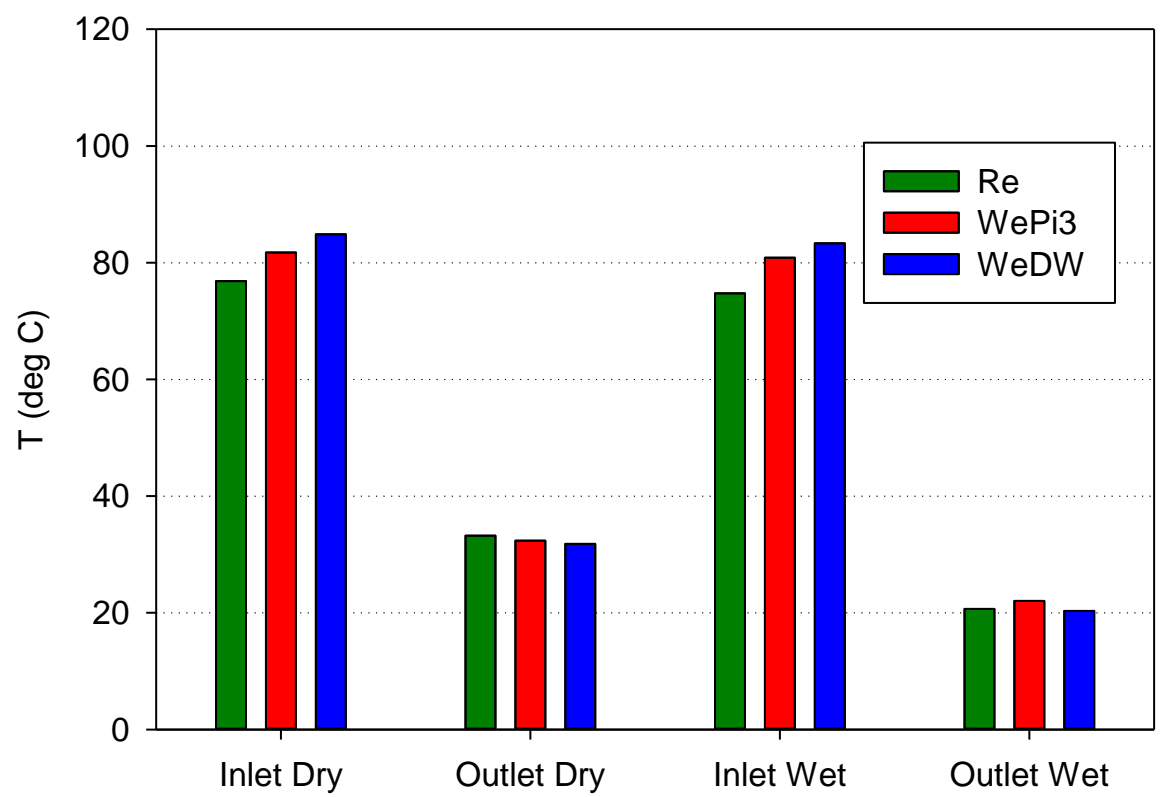

Heated Air Energy Input

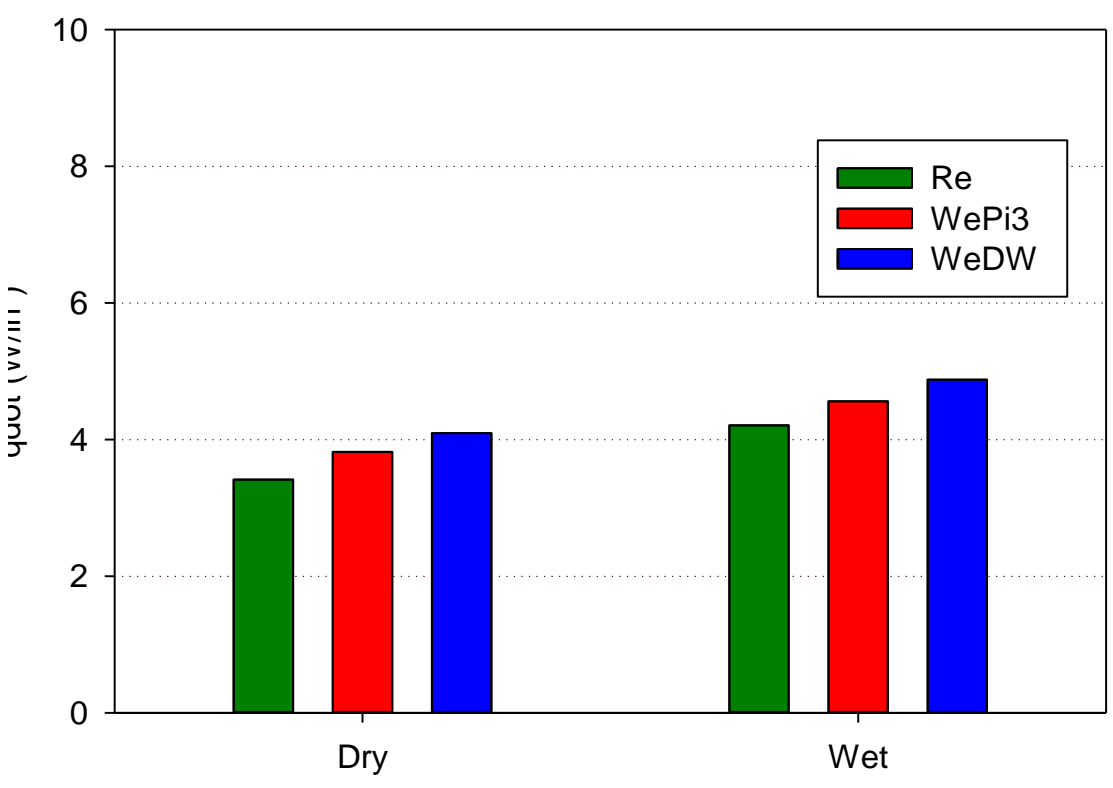




\section{Warm Hold Scenario}

\begin{tabular}{|cccccccccccc|}
\hline $\begin{array}{c}\text { Scale } \\
\text { Method }\end{array}$ & Alt (ft) & $V(\mathrm{kts})$ & $\begin{array}{c}\mathrm{T}_{\mathrm{s}} \\
(\mathrm{deg} \mathrm{C})\end{array}$ & $\begin{array}{c}\mathrm{LWC} \\
\left(\mathrm{g} / \mathrm{m}^{3}\right)\end{array}$ & $\begin{array}{c}\mathrm{MVD} \\
(\mu \mathrm{m})\end{array}$ & $\begin{array}{c}\mathrm{m}_{\mathrm{w}} \\
\left(\mathrm{g} / \mathrm{m}^{2} \mathrm{~s}\right)\end{array}$ & $\begin{array}{c}\mathrm{Re}_{2 \mathrm{r}} \\
\left(\mathrm{x} 10^{6}\right)\end{array}$ & WeDA & Pi3 & $\begin{array}{c}\text { WeDW } \\
\left(\mathrm{x} 10^{6}\right)\end{array}$ & $\begin{array}{c}\text { lce Mass } \\
(\mathrm{g})\end{array}$ \\
\hline Reference & 15000 & 185 & -8.6 & 0.39 & 18.3 & 20.0 & 0.186 & 4922 & 1.74 & 6.55 & N/A \\
Re & 1336 & 109 & -6.3 & 0.66 & 25.4 & 20.0 & 0.186 & 2923 & 3.09 & 2.26 & 207.5 \\
WePi3 & 1814 & 147 & -7.3 & 0.36 & 19.6 & 13.6 & 0.241 & 4922 & 1.74 & 4.12 & 64.5 \\
WeDW & 2454 & 184 & -8.6 & 0.37 & 22 & 20.0 & 0.299 & 8005 & 2.24 & 6.55 & 138.8 \\
\hline
\end{tabular}

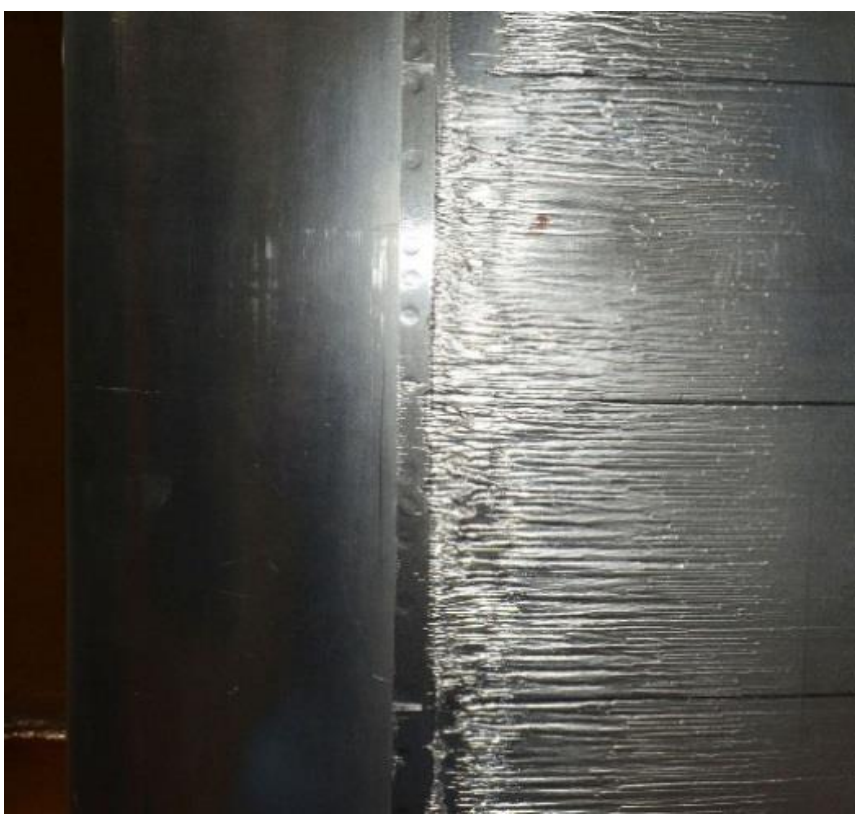

Re Scaling

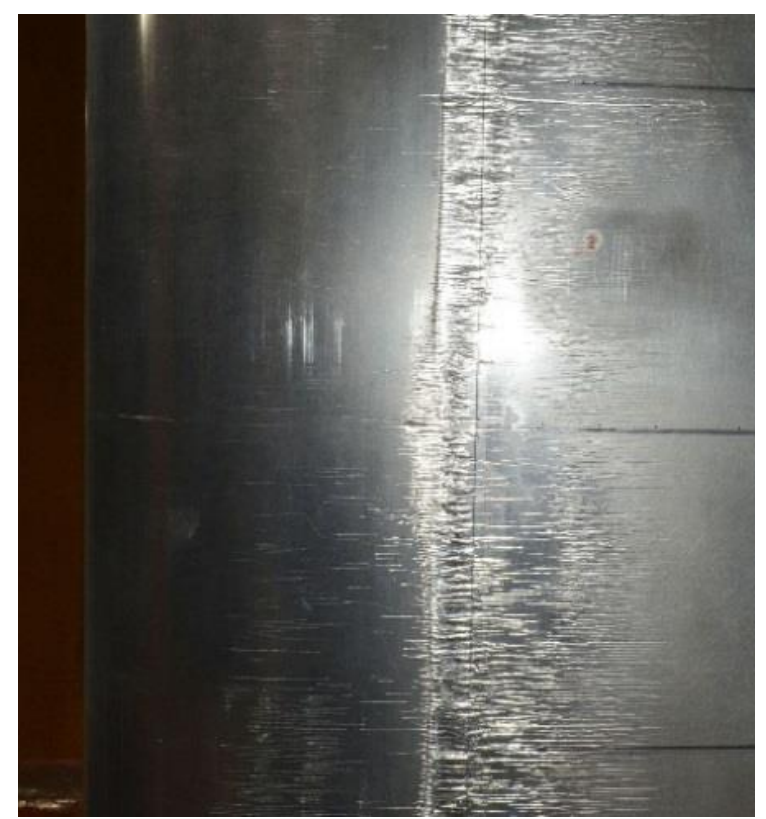

WePi3 Scaling

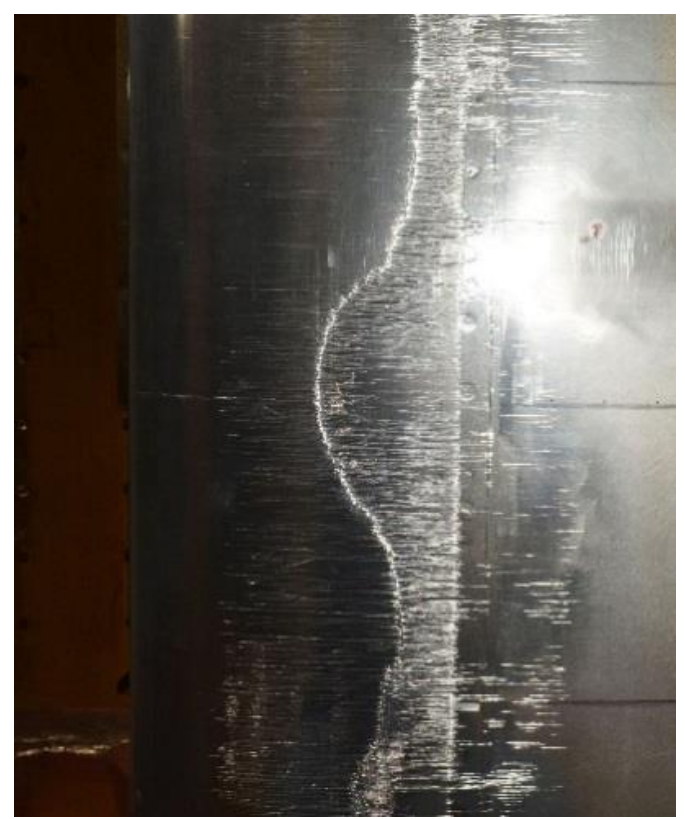

WeDW Scaling 


\section{Warm Hold Scenario}

\section{LE Surface Temperature}

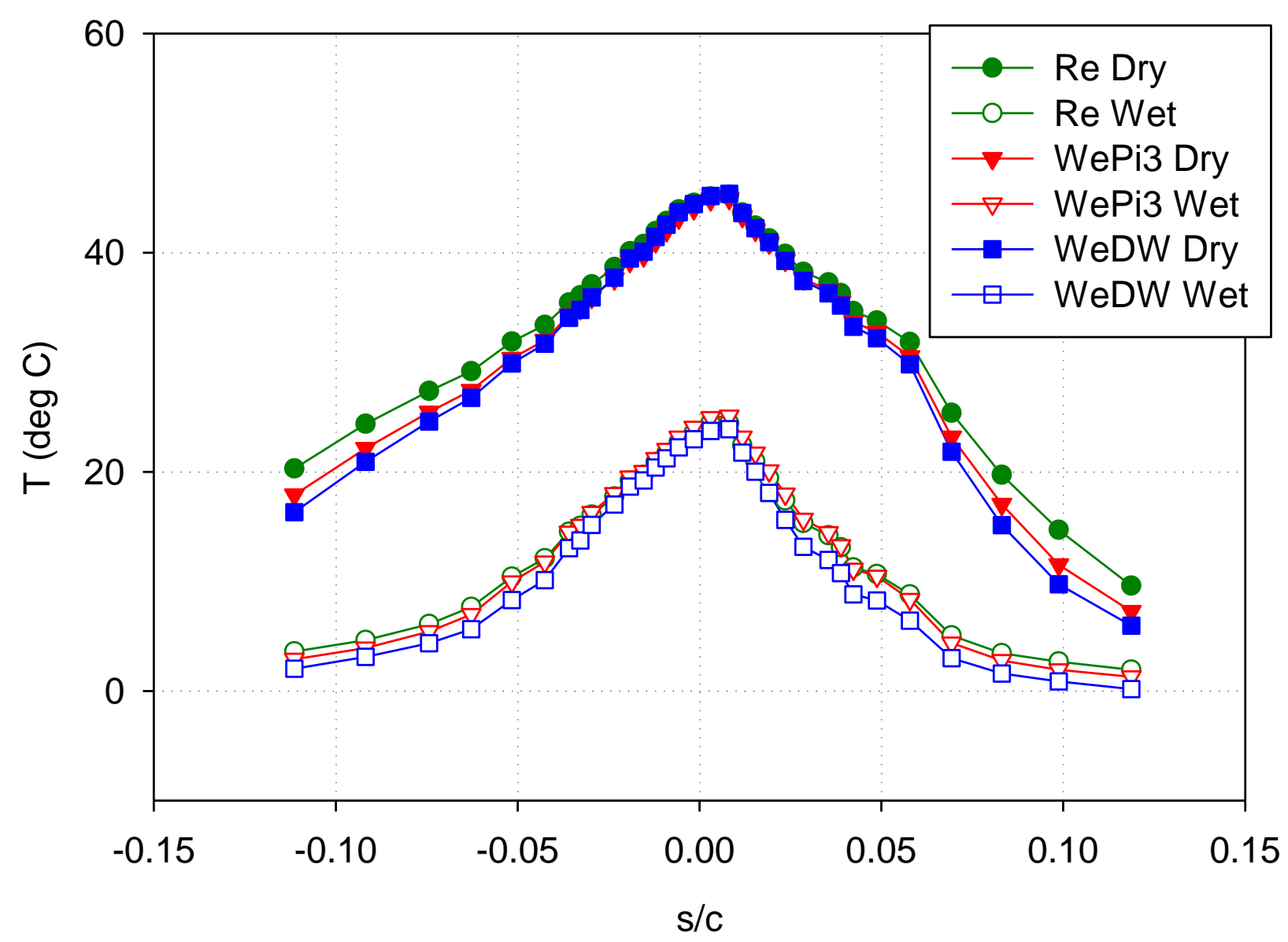




\section{Warm Hold Scenario}

Heated Air Temp.

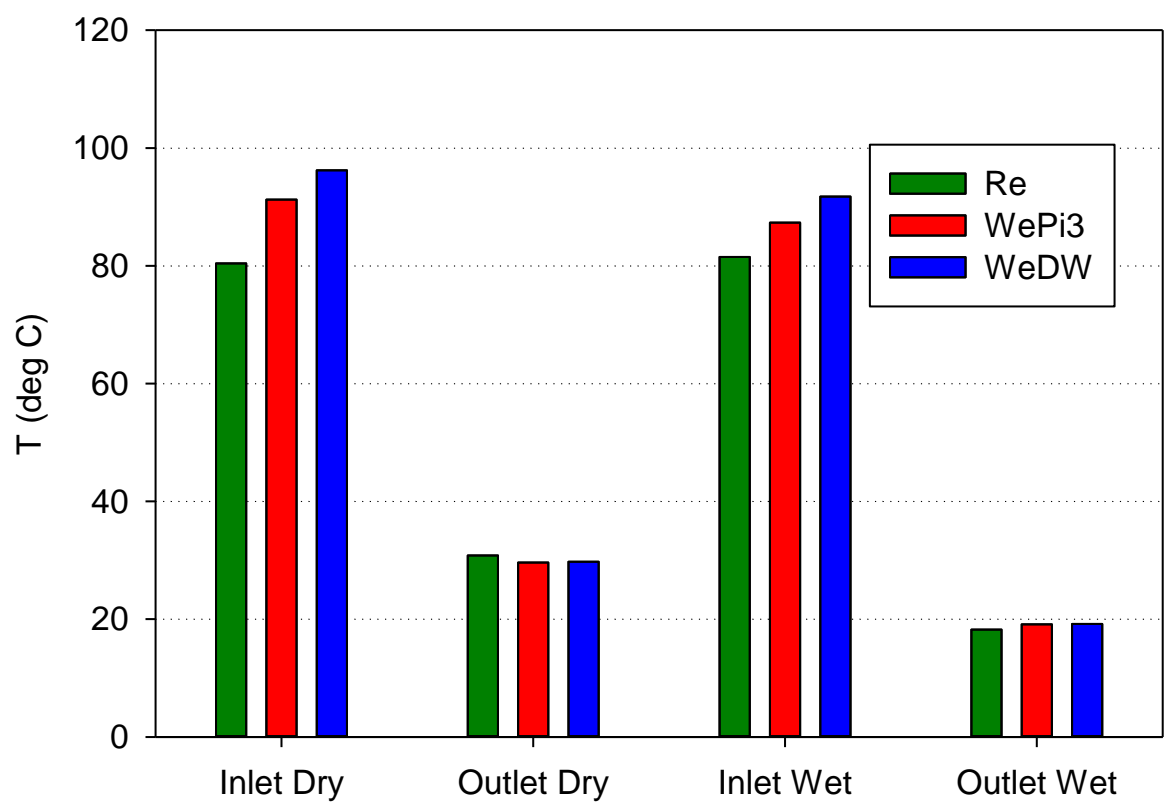

Heated Air Energy Input

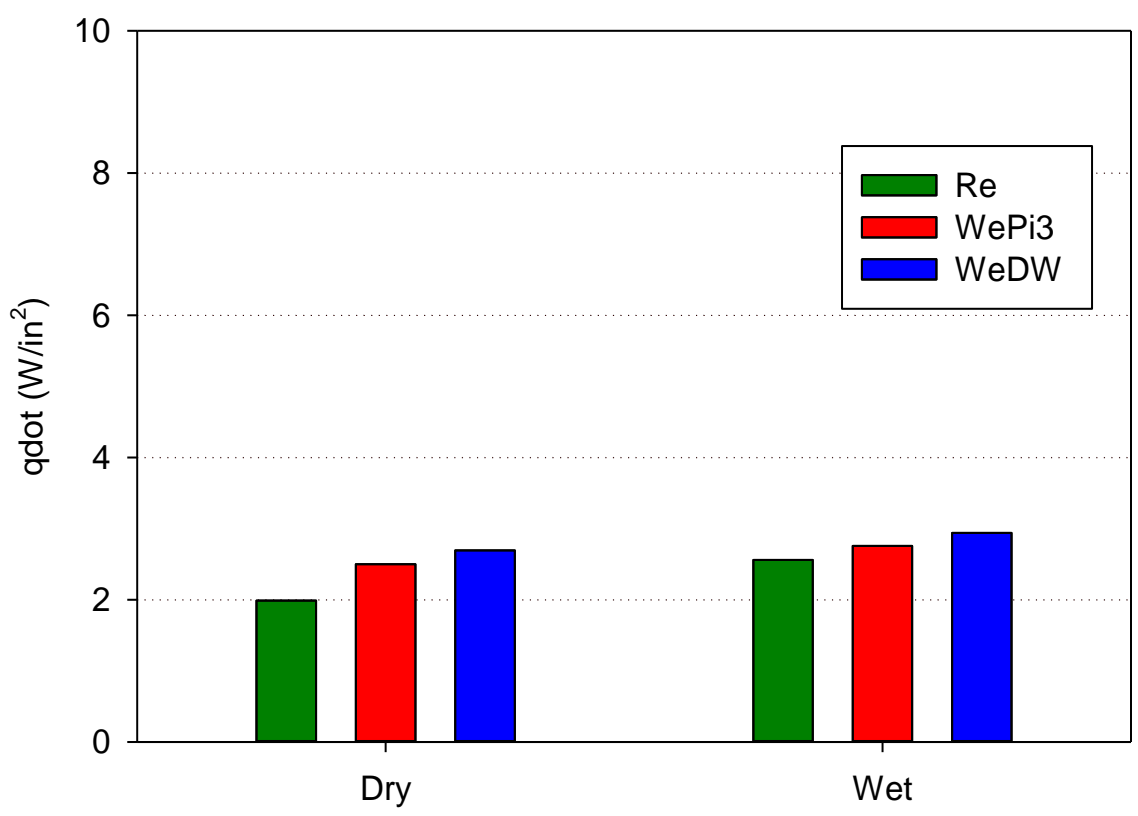




\section{Conclusion}

- Test conducted at NASA Icing Research Tunnel to evaluate new altitude scaling methods for thermal ice protection systems.

- Two Weber number-based scaled methods developed during a series of joint NASA and NRCC tests at AIWT.

- Results from IRT generally agreed with and supported the results from previous tests in NRCC.

- We-based scaling methods resulted in smaller ice accretion that formed farther upstream than the Re-based scaling methods.

- Additional tests required in altitude capable tunnels using fullscale models to better define the limits of physical relationships used to develop these scaling methods. 


\section{Extra Slides}




\section{WSU Warm Hold Scenario}

\begin{tabular}{|cccccccccccc|}
\hline $\begin{array}{c}\text { Scale } \\
\text { Method }\end{array}$ & Alt (ft) & $V(\mathrm{kts})$ & $\begin{array}{c}\mathrm{T}_{\mathrm{s}} \\
(\mathrm{deg} \mathrm{C})\end{array}$ & $\begin{array}{c}\mathrm{LWC} \\
\left(\mathrm{g} / \mathrm{m}^{3}\right)\end{array}$ & $\begin{array}{c}\mathrm{MVD} \\
(\mu \mathrm{m})\end{array}$ & $\begin{array}{c}\mathrm{m}_{\mathrm{w}} \\
\left(\mathrm{g} / \mathrm{m}^{2} \mathrm{~s}\right)\end{array}$ & $\begin{array}{c}\mathrm{Re}_{2 \mathrm{r}} \\
\left(\mathrm{x} 10^{6}\right)\end{array}$ & WeDA & $\mathrm{Pi3}$ & $\begin{array}{c}\text { WeDW } \\
\left(\mathrm{x} 10^{6}\right)\end{array}$ & $\begin{array}{c}\text { Ice Mass } \\
(\mathrm{g})\end{array}$ \\
\hline Reference & 15000 & 205 & -9.4 & 0.5 & 20 & 31.1 & 0.205 & 6065 & 1.73 & 8.04 & N/A \\
Re & 1312 & 126 & -6.3 & 0.82 & 27.2 & 31.1 & 0.205 & 3769 & 2.98 & 3.01 & 236.5 \\
WePi3 & 1835 & 164 & -7.6 & 0.43 & 24 & 21.0 & 0.264 & 6065 & 1.73 & 5.13 & 68.9 \\
WeDW & 2446 & 205 & -9.2 & 0.5 & 22.4 & 31.1 & 0.324 & 9715 & 2.23 & 8.04 & 266.2 \\
WSU & 1191 & 115 & -9.4 & 0.87 & 29 & 30.8 & 0.192 & 3229 & 3.03 & 2.53 & 483.3 \\
\hline
\end{tabular}

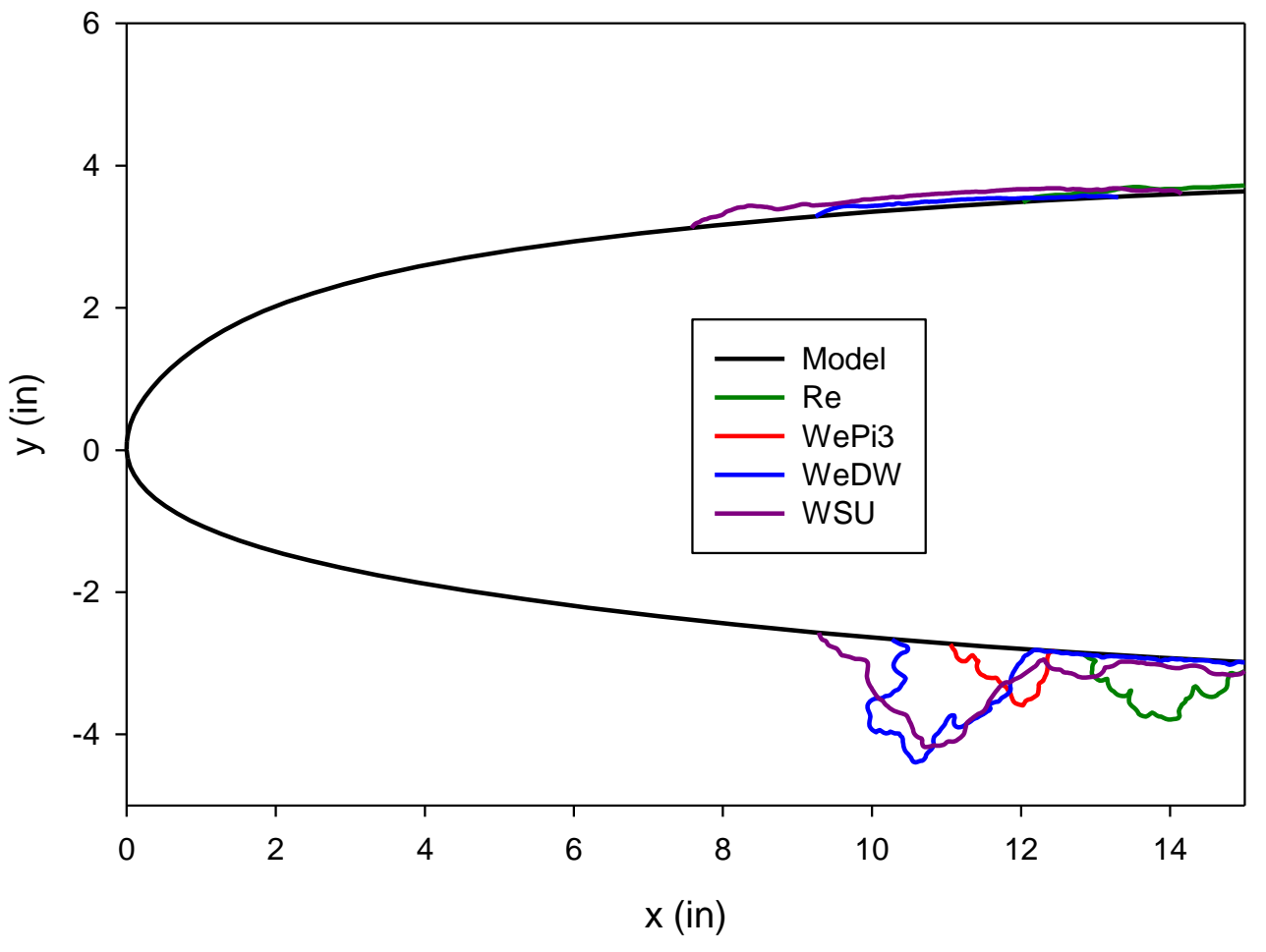




\section{WSU Warm Hold Scenario}

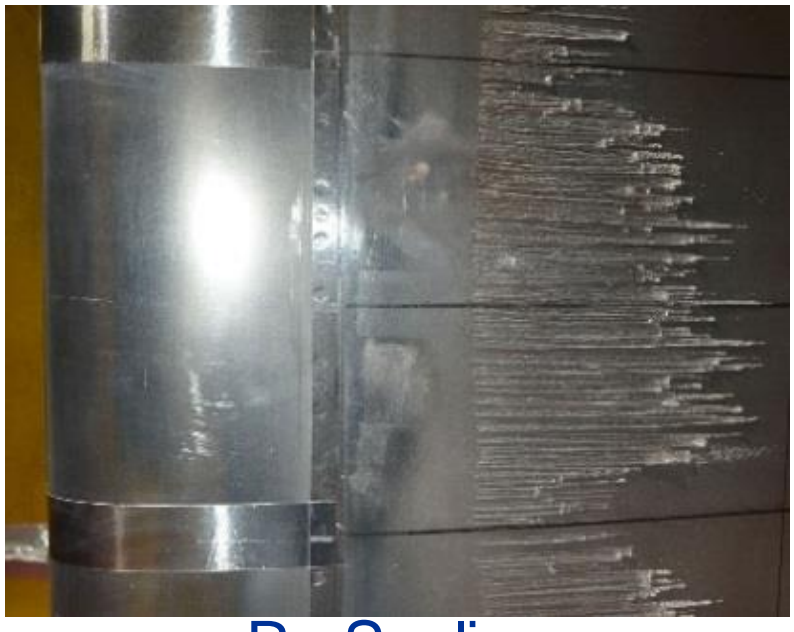

Re Scaling

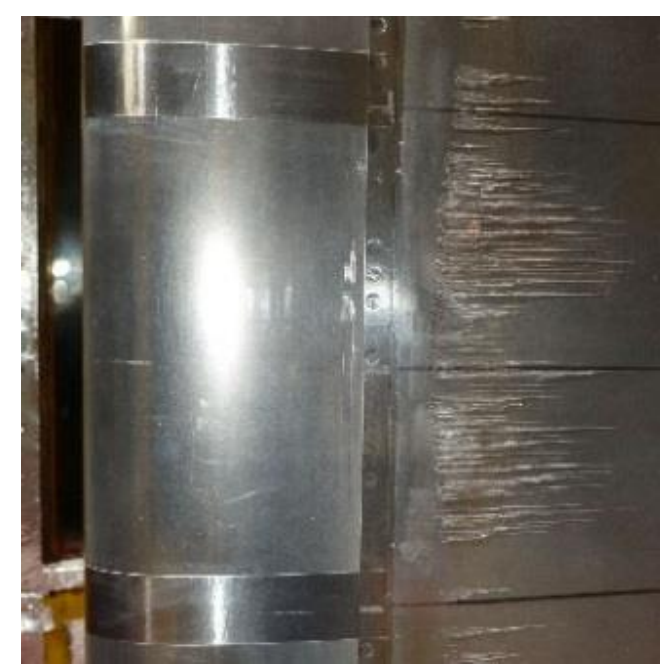

WeDW Scaling

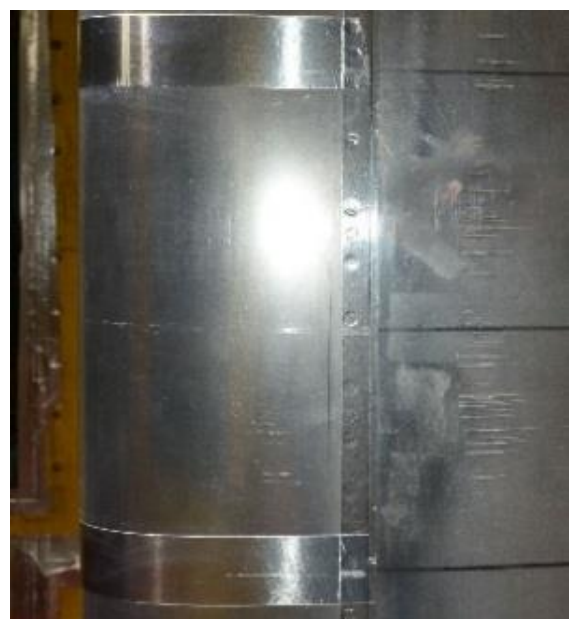

WePi3 Scaling

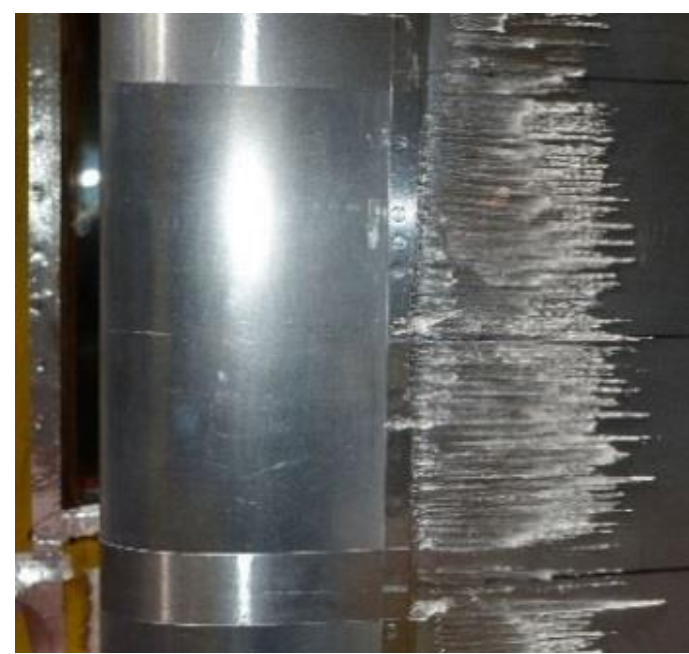

WSU Scaling 


\section{WSU Warm Hold Scenario}

\section{LE Surface Temp.}

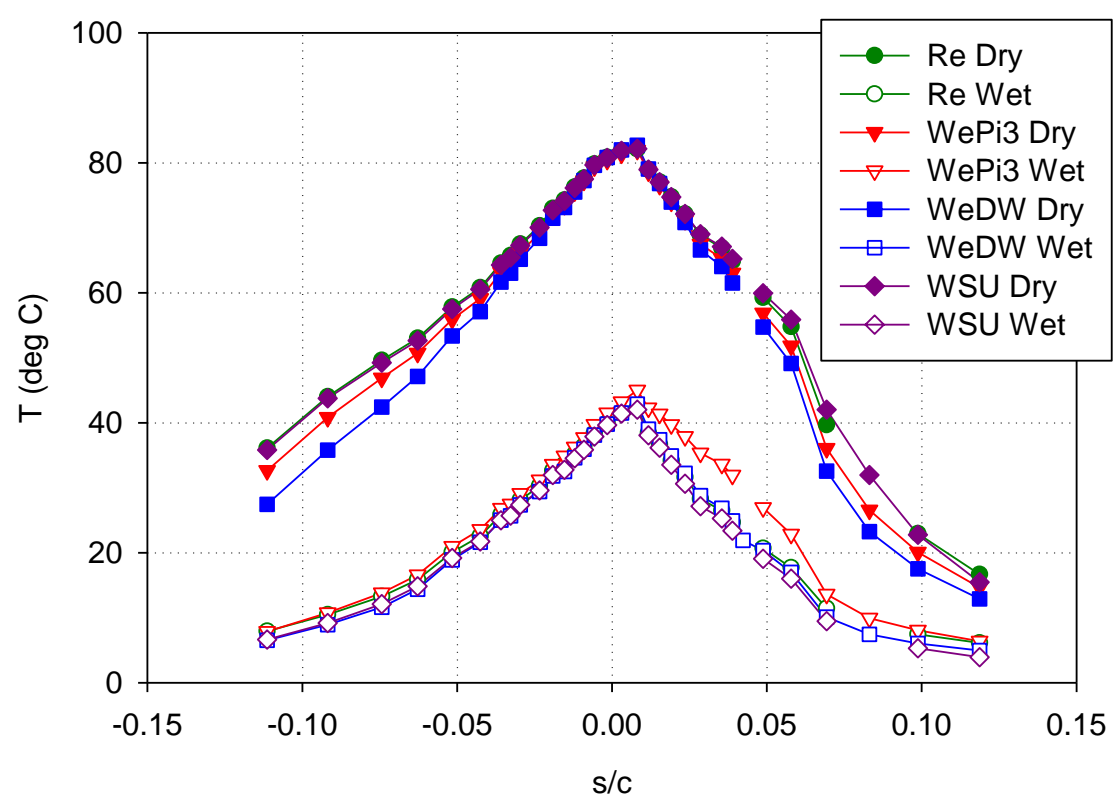

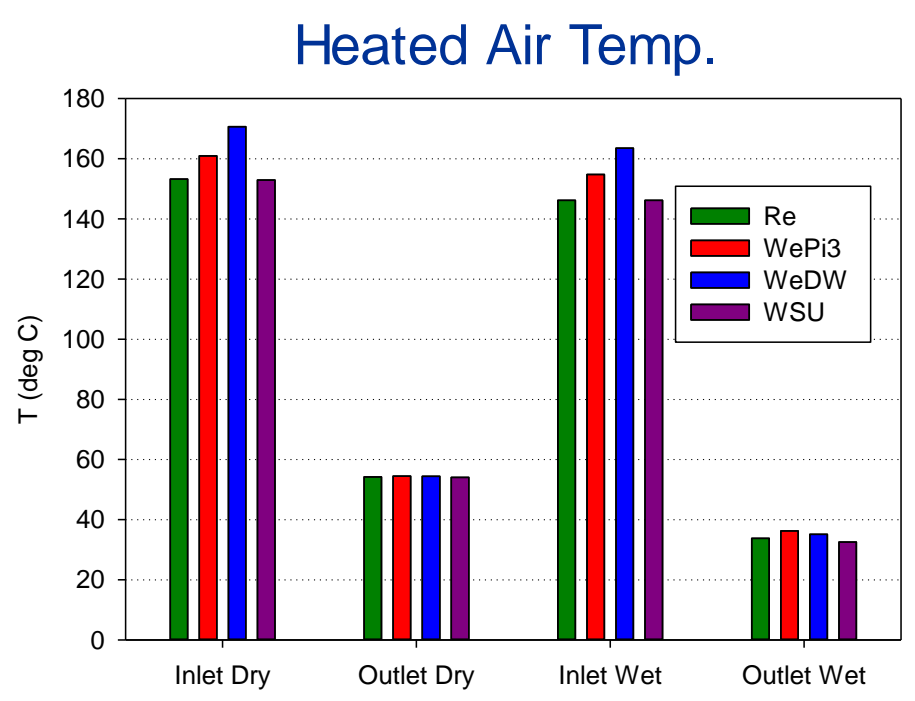

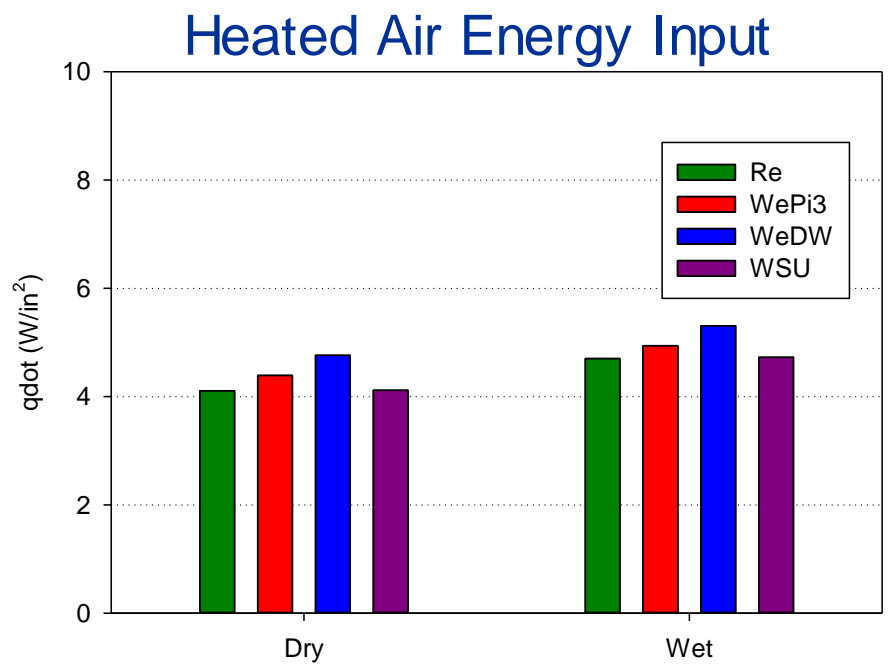

